\title{
Traduire
}

Une autre perspective sur r tr traduction

Revue française de la traduction

$222 \mid 2010$

Traduire pour le théâtre

\section{Une histoire de la langue de bois}

\section{Bernhard Lorenz}

\section{(2) OpenEdition \\ Journals}

Édition électronique

URL : http://journals.openedition.org/traduire/455

DOI : 10.4000/traduire.455

ISSN : 2272-9992

\section{Éditeur}

Société française des traducteurs

\section{Édition imprimée}

Date de publication : 15 juin 2010

Pagination : 145-148

ISSN : 0395-773X

\section{Référence électronique}

Bernhard Lorenz, «Une histoire de la langue de bois », Traduire [En ligne], 222 | 2010, mis en ligne le 12 novembre 2013, consulté le 22 septembre 2020. URL : http://journals.openedition.org/traduire/455 ; DOI : https://doi.org/10.4000/traduire.455 


\section{Compte rendu d'ouvrage Une histoire de la langue de bois}

\section{Bernhard Lorenz}

La sagesse, le bon sens, l'intérêt du gouvernement, c'est d'être constamment tonifié par une opposition sérieuse, solide, constructive (J. Lang).

Théâtre et politique - même combat. La scène politique peut être vue comme l'arène du monde politique en action, et les acteurs politiques sont les acteurs de ce théâtre et jouent les têtes d'affiche, ou plutôt, les têtes de liste. Ces acteurs ne sont pourtant rien sans les mots, sans un discours dont les paroles enjolivent, cachent la réalité, la modifient pour s'approcher parfois de l'hypocrisie, dont l'origine grecque Hupokrisis signifie " jeu d'acteur ".

Traducteurs, traductrices, je vais là vous donner le fond de ma pensée, rien que ma vérité. En 13 chapitres (j'en ai lu un par jour), sur quelque 342 pages, dont 20 pour les sources et la bibliographie, Christian Leporte, spécialiste de l'histoire des médias et de la communication politique, nous brosse le tableau d'une histoire de la langue de bois. II nous emmène faire un tour de piste des stratégies idéologiques et tactiques politiques basées sur la seule langue. Je l'ai dévoré ce livre, devenu pour moi l'ébauche d'une histoire du mensonge et de la sincérité, ainsi que des propagandes et de la communication politique et économique.

Pourquoi en parler dans une publication pour traducteurs ? Nous sommes tous des citoyens et pouvons puiser dans ces pages de manière plaisante des ressources pour mieux appréhender les discours et subterfuges du discours, non seulement celui des politiciens et des sorciers de la communication financière, mais également celui des agences immobilières (qui n'a pas déjà vu d'annonces pour un " coquet studio " ?).

Donc, pas un livre sur un thème de la traduction, mais un voyage à travers une des contrées de la langue française qui a ses frères et sœurs dans toutes les langues, comme le montre la liste évocatrice égrenée au début du livre : "langue de drap " ou "langue de chêne " (Russie tsariste), "langue herbeuse ", "langue engourdie ", " novlangue " (newspeak), "langue de caoutchouc ", "langue de béton " (Betonsprache) ou Apparatschiksprache (langue des apparatchiks), guan qiang (ton du mandarin), le tecque-tecque (le " tac-tac " des mots) (Cuba), Gobbledegook, double-speak ou double-talk, "discours de comices agricoles " (Flaubert), 
" sinolangue " ou « nazilangue ", ou le mot entendu à la radio après la qualification de la France pour le Mondial du foot : "langue de cuir " de R. Domenech, la "langue de miel " de Mme BruniSarkozy ou encore ses cousins en Europe tels qu'" eurolangue ", " eurobabillage ", eurokauderwelsch, eurospeak, eurofog...

Une des autres raisons qui m'incite à vous inviter à la lecture de ce livre est le rappel de G. Orwell et de ses règles d'or qui, à mon sens, ont une place de choix dans notre travail, et auxquelles Delporte fait souvent référence : " s'il est possible de supprimer un mot, supprimezle toujours [...] n'utilisez jamais le passif si vous pouvez utiliser l'actif... n'utilisez jamais un mot long quand un court convient [...]".

Delporte situe la naissance de la langue de bois vers 1790 ("Dictionnaire national et anecdotique "(P.N. Chantreau)). Le Trésor de la langue Française la définit ainsi : "Manière d'exprimer sous une forme codée, dans une phraséologie stéréotypée et dogmatique, à l'aide d'euphémismes, de lieux communs, de termes généraux et/ou abstraits, un message idéologique qui ne sera pas opposable à ses auteurs, mais qui cependant sera compris dans son vrai sens par un petit nombre d'initiés ; p. ext. tout langage qui s'alimente au dictionnaire des idées et des formes reçues."

Les caractéristiques de la langue de bois selon Delporte sont l'euphémisation, la relativisation, la minimisation, le flou volontaire du vocabulaire (" évidence manifeste ") parfois grâce au

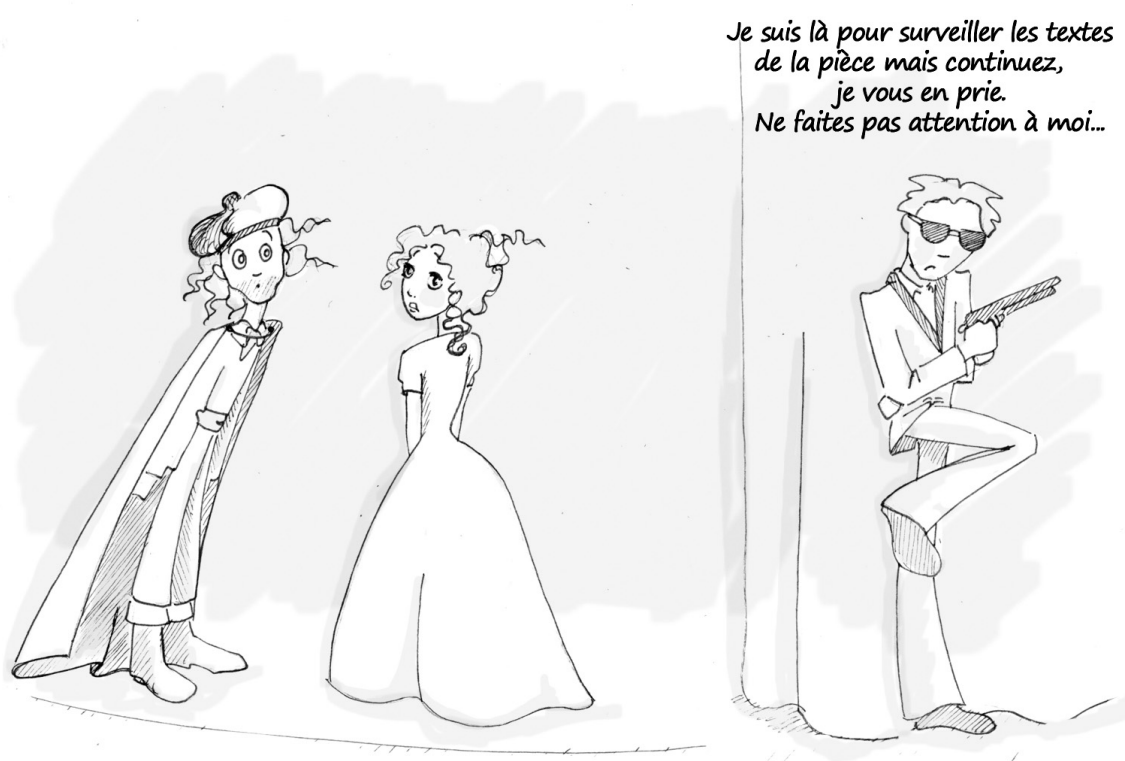


recours à une certaine lourdeur ("réaliser/créer les conditions "), l'omission et la formule floue. Tournures immuables dans lesquelles le nom ou le verbe ne prennent leur sens que soulignés par un adjectif ou un adverbe : "la force décisive de la classe ouvrière ", le "bilan globalement positif ". Elle accole un terme glorieux qui efface toute neutralité sémantique, ex. "la révolution " devient "le triomphe de la révolution".

Parfois, le flou est organisé avec le but de contrôler sa parole et de dresser une sorte d'écran protecteur entre l'opinion publique et soi. Ainsi, dans le discours officiel on n'échoue jamais : on est confronté à des problèmes, à des obstacles, à des situations complexes, on subit des difficultés ou des complications. Un syndicat n'enregistre pas de baisse de son effectif, il n'y aura que " consolidations ", " tassements ", " retards ". Un parti politique n'est jamais en crise ou divisé : il connaît juste " des divergences ". Quant aux succès au front d'une guerre (réelle, financière, contre la maladie) : il n'y a pas d'immobilisation, de recul ou de retraite ; par contre, on assiste à une "guerre de défense mobile ", les défaites deviennent " des revers ", la fuite se mue en "dégagement " ou " repli tactique".

Delporte, jamais ennuyeux, nous balade ainsi de la Terreur à la République des verbomanes, en passant par la sovietlangue et les bourreurs de crânes (de $B$ comme Bonaparte à $B$ comme Bush). II passe en revue des " sujets brûlants " (actualité) ainsi que les formules utilisées pendant la Crise financière de 2007-2009 (ex. : naissance de la " croissance négative " - donc un PIB qui augmente en diminuant ou croît en décroissant...), fait un tour du côté de " la rigueur " et de "l'austérité " qui mène à la " rigueur pour la modernisation ". II trace le chemin parcouru du " Je dirai tout "d'un Napoléon qui manie le premier la langue de bois moderne en temps de guerre jusqu'à la Sarkolangue ("le Parler vrai " $\rightarrow$ je parle comme vous $=$ je suis comme vous = vous pouvez me faire confiance).

Les spécificités de la langue de bois en Afrique et Cuba ne sont pas oubliées non plus (j'ai souri à la lecture de l'exemple : "Défense de marcher sur la pelouse " (France) " traduit "à Cuba par "Un peuple cultivé prend soin de ses espaces verts "!

Delporte m'a fait sourire aussi avec son « Guide à l'usage des apparatchiks débutants pour un discours universel " (reproduit par Libération dans son édition des 4 et 5 juillet 1981). II signale le "générateur de langue de bois " (Tunezine - 2004), p. 183, ainsi que le " générateur de l'ENA-langue ". Sans oublier son tour de piste des mots nouveaux, nés sous l'influence du politiquement correct tels que "l'aveugle " qui devient " non-voyant " puis " handicapé visuel " ou " déficient visuel ", le " camping " devenu " hôtellerie de plein air ", le " mensonge " une " contre-vérité "...

Avec de nombreux extraits d'interviews à l'appui, Delporte démontre aussi que dès les années 1970, la langue de bois sera modelée pour la télévision (ses images, ses émissions, son format) - avec comme conséquence un appauvrissement sémantique ou l'avènement des "Sound bites " (inserts sonores - petites phrases conçues pour des spots télévisés de quelques 
secondes). Ce qui a ensuite conduit à l'» invention " de la petite phrase qui se retient, utilisable à toutes les sauces après : "le monopole du cœur " (V. Giscard d'Estaing), "I'homme du passé qui devient l'homme du passif ", "La crise du développement est le développement de la crise " (Chirac 2002).

Chaque page de ce livre fourmille de trouvailles, de citations, de remarques qui font mouche. L'écriture de Delporte est allègre, jamais indigeste, et on se sent plus intelligent après l'avoir lu. Certes, il y a un côté "Caverne d'Ali Baba " (un peu comme ma fiche de lecture, non ?) et pour ceux qui préfèrent une lecture plus ardue, universitaire, la bibliographie fourmille d'invitations à un rendez-vous avec cette émanation de la communication si particulière.

Une histoire de la langue de bois, Christian DELPORTE, Éditions Flammarion, Paris, 2009, 352 pages, $21 €$. ISBN : 978-2-0812-1993-9

Christian Delporte est professeur d'histoire contemporaine à l'université de Versailles-SaintQuentin-en-Yvelines. II a écrit de nombreux ouvrages comme dernièrement le Dictionnaire d'histoire culturelle de la France contemporaine (PUF, 2010). 